
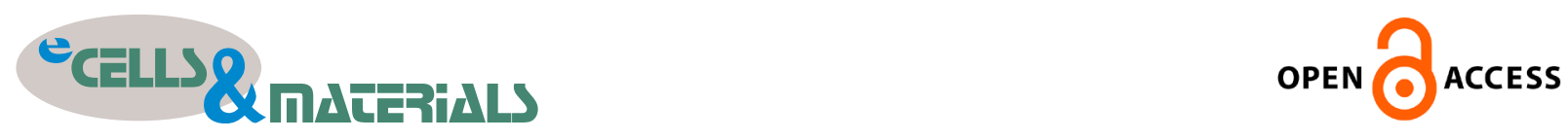

\title{
MESENCHYMAL STEM CELL SECRETOME REDUCES PAIN AND PREVENTS CARTILAGE DAMAGE IN A MURINE OSTEOARTHRITIS MODEL
}

\author{
S. Khatab ${ }^{1,2}$, G.J.V.M. van Osch ${ }^{1,3, *}$, N. Kops ${ }^{1}$, Y.M. Bastiaansen-Jenniskens ${ }^{1}$, P.K. Bos ${ }^{1}$, J.A.N. Verhaar ${ }^{1}$, \\ M.R. Bernsen² and G.M. van Buul ${ }^{1}$ \\ ${ }^{1}$ Department of Orthopaedics, Erasmus MC, Rotterdam, the Netherlands \\ ${ }^{2}$ Department of Radiology and Nuclear Medicine, Erasmus MC, Rotterdam, the Netherlands \\ ${ }^{3}$ Department of Otorhinolaryngology, Erasmus MC, Rotterdam, the Netherlands
}

\begin{abstract}
Mesenchymal stem cells (MSCs) represent a promising biological therapeutic option as an osteoarthritis (OA)-modifying treatment. MSCs secrete factors that can counteract inflammatory and catabolic processes and attract endogenous repair cells. The effects of intra-articular injection of MSC secretome on OA-related pain, cartilage damage, subchondral bone alterations and synovial inflammation were studied in a mouse collagenase-induced OA model. The MSC secretome was generated by stimulating human bone-marrowderived MSCs with interferon gamma (IFN $\gamma$ ) and tumour necrosis factor alpha (TNF $\alpha) .54$ mice were randomly assigned to injections with i) MSC secretome from 20,000 MSCs, ii) 20,000 MSCs or iii) medium (control). Pain was assessed by hind limb weight distribution. Cartilage damage, subchondral bone volume and synovial inflammation were evaluated by histology. MSC-secretome- and MSC-injected mice showed pain reduction at day 7 when compared to control mice. Cartilage damage was more abundant in the control group as compared to healthy knees, a difference which was not found in knees treated with MSC secretome or MSCs. No effects were observed regarding synovial inflammation, subchondral bone volume or the presence of different macrophage subtypes. Injection of MSC secretome, similarly to injection of MSCs, resulted in early pain reduction and had a protective effect on the development of cartilage damage in a murine OA model. By using the regenerative capacities of the MSC-secreted factors, it will be possible to greatly enhance the standardisation, affordability and clinical translatability of the approach. This way, this biological therapy could evolve towards a true disease-modifying anti-osteoarthritic drug.
\end{abstract}

Keywords: Mesenchymal stem cell, secretome, osteoarthritis, animal model, pain, synovial inflammation, cartilage damage.

*Corresponding author: Prof. Gerjo J.V.M. van Osch, PhD, Department of Orthopaedics and Otorhinolaryngology, Erasmus MC, University Medical Centre, Wytemaweg 80, 3015 CN Rotterdam, the Netherlands.

Telephone number: +31 107043661 Email: g.vanosch@erasmusmc.nl

Copyright policy: This article is distributed in accordance with Creative Commons Attribution Licence (http://creativecommons.org/licenses/by-sa/4.0/).

\section{Introduction}

Osteoarthritis (OA) is a disabling joint disease affecting over $10 \%$ of individuals from the age of 60 (Zhang and Jordan, 2010). OA is characterised by loss of cartilage integrity, subchondral bone changes, formation of osteophytes and inflammation of the synovial membrane (Berenbaum et al., 2013). The interplay between these processes and tissues and their exact role in the aetiology and progression of the disease is yet unclear. These processes together result in pain and functional disability, which are the main reasons for patients to seek medical treatment. To this date, no curative treatment for OA exists. Current treatments mainly aim at the treatment of the disease symptoms and not at a durable way of modifying pathological osteoarthritic processes. Current therapeutic options include life-style changes, physical therapy, pain medication and for end-stage OA - joint replacement. Since joint arthroplasties have a limited life-span, the need for disease-modifying drugs or therapies is high. Ideally, 
such a therapy would inhibit or repair damage to the joint tissues and simultaneously reduce pain and disability.

Mesenchymal stem cells (MSCs) represent a promising biological therapeutic option as an OAmodifying treatment. Stem cells play a pivotal role in physiological tissue homeostasis and regeneration after tissue injury (Caplan and Dennis, 2006). Next to their differentiation potential into several lineages, including the chondrogenic lineage, MSCs can influence their (micro)environment by secreting trophic mediators (Caplan, 2017; Kinnaird et al., 2004; Le Blanc and Mougiakakos, 2012; Prockop, 2009; Ren et al., 2008; Timmers et al., 2011). These secreted factors could possibly counteract inflammatory and catabolic processes and attract endogenous repair cells (Estrada et al., 2009; Prockop and Oh, 2012; Timmers et al., 2011; van Buul et al., 2012). Several (pre)clinical studies show promising results for intra-articular stem cell injection as a treatment for OA (Gupta et al., 2016; Lamo-Espinosa et al., 2016; Murphy et al., 2003; Pers et al., 2016; Ter Huurne et al., 2012; van Buul et al., 2014). Murphy et al. (2003) are the first to show amelioration of degenerative changes after intra-articular injection of bone marrow-derived MSCs in a caprine OA model. Others find diminishment of OA-derived pain (van Buul et al., 2014) or synovial inflammation and cartilage degradation in pre-clinical studies (Ter Huurne et al., 2012). Initial reports from clinical studies indicate that intra-articular application of MSCs is safe for OA and possibly results in clinical improvement (Gupta et al., 2016; Pers et al., 2016).

The MSC trophic capacities provide the interesting option of possibly basing future therapies on the secreted factors rather than on the MSCs themselves. The importance of the endocrine MSC function is further endorsed by the fact that locally- (van Buul et al., 2014) or systemically- (Eggenhofer et al., 2014) applied MSCs show very limited long-term engraftment, for which a 'hit and run' mechanism is postulated (Prockop, 2009; von Bahr et al., 2012). In addition, the MSCs need to be activated to exert their trophic effects (Barrachina et al., 2017; Crop et al., 2010; van Buul et al., 2012). Such an in vivo activation is difficult to control or influence and, thereby, likely to be subject to large variability. In vitro stimulation with, for instance, inflammatory factors provides an option to further optimise the use of the MSC immunomodulatory abilities.

The use of the MSC secretome could improve the therapeutic efficacy and would greatly enhance the clinical applicability of this biological treatment as a true disease-modifying anti-osteoarthritic drug. The aim of the present study was to explore the antiosteoarthritic effects of the human MSC secretome, compared to the MSCs, on various outcome measures in a collagenase mouse OA model. Injection of human MSC secretome was hypothesised to be at least as effective as MSCs in reducing OA-related pain, cartilage damage, subchondral bone alterations and synovial inflammation.

\section{Materials and Methods}

\section{Expansion of MSCs}

Human bone marrow MSCs were derived from heparinised femoral-shaft marrow aspirate of six patients undergoing total hip replacement (mean age $55.3 \pm 10.0$ years; female : male ratio $1: 2$ ) using previously described procedures (de Mos et al., 2007), after written informed consent and approval by the medical ethical committee (protocol METC-2004-142). Briefly, bone marrow cells were plated at 50,000 cells/ $\mathrm{cm}^{2}$ and, after $24 \mathrm{~h}$, the flasks were washed to remove non-adherent cells. Then, MSCs were trypsinised [0.25\% trypsin/ethylenediaminetetraacetic acid (EDTA) solution (Life Technologies)] and seeded in cell culturing flasks at a density of 2,300 cells/ $\mathrm{cm}^{2}$ in expansion medium consisting of minimal essential medium alpha ( $\alpha$ MEM; Gibco), $10 \%$ heatinactivated foetal calf serum (FCS; Gibco), $1.5 \mu \mathrm{g} /$ $\mathrm{mL}$ fungizone (Invitrogen), $50 \mu \mathrm{g} / \mathrm{mL}$ gentamicin (Invitrogen), $25 \mu \mathrm{g} / \mathrm{mL}$ ascorbic acid-2-phosphate (Sigma-Aldrich) and $1 \mathrm{ng} / \mathrm{mL}$ fibroblast growth factor 2 (FGF2; AbD Serotec, Oxford, UK). Cells were cultured in an incubator at $37{ }^{\circ} \mathrm{C}, 5 \% \mathrm{CO}_{2}$ and $90 \%$ humidity. Medium was refreshed twice a week. MSCs were passaged at approximately $70 \%$ confluency. MSCs used for in vivo experiments were characterised by fluorescence-activated cell sorting (FACS) analysis and resulted positive for CD73, CD90, CD105 and CD166 and negative for CD11C, CD31 and CD45 (data not shown). de Mos et al. (2007) show tri-lineage differentiation of cells isolated following this procedure. The viability of the MSCs was evaluated after trypsinisation and before being injected or seeded for obtaining the secretome: less than $5 \%$ of the cells were dead, as indicated by trypan blue positive staining. Human MSCs from endstage OA donors were used to increase the clinical translatability of the study.

\section{Preparation of MSC secretome}

To produce the MSC secretome, passage 3 MSCs were plated at a density of $3.5 \times 10^{4}$ cells $/ \mathrm{cm}^{2}$ and cultured for $24 \mathrm{~h}$ in expansion medium. After $24 \mathrm{~h}$, cells were activated to secrete immunomodulatory factors by culturing for $24 \mathrm{~h}$ in stimulating medium (van Buul et al., 2012). This stimulating medium consisted of $\alpha$ MEM supplemented with $1.5 \mu \mathrm{g} / \mathrm{mL}$ fungizone, $50 \mu \mathrm{g} / \mathrm{mL}$ gentamicin, $1 \%$ insulin-transferrinselenium (ITS; Biosciences), $50 \mathrm{ng} / \mathrm{mL}$ interferon gamma (IFN $\gamma$; PeproTech) and $50 \mathrm{ng} / \mathrm{mL}$ tumour necrosis factor alpha (TNF $\alpha$; PeproTech). After $24 \mathrm{~h}$ of stimulation, MSCs were washed five times with phosphate-buffered saline (PBS; Gibco). To collect the paracrine factors, collecting medium was added, consisting of only $\alpha \mathrm{MEM}$ with $0.05 \%$ bovine 
serum albumin (BSA; Sigma-Aldrich) - to stabilise the secreted factors and as an adhesive for smaller molecules to bind to and to be retained after the concentration step - and without phenol red - which can mimic an oestrogen and, therefore, influence cell behaviour in vivo. $1 \mathrm{~mL}$ of collecting medium was added per $2.0 \times 10^{5}$ MSCs. MSC secretome was collected after $24 \mathrm{~h}$ and centrifuged at $700 \times g$ for $8 \mathrm{~min}$ to remove cell debris. The secretome equivalent of 20,000 MSCs, a number of cells previously used in a mouse collagenase OA model (Ter Huurne et al., 2012), was planned to be injected in a murine knee joint. To achieve this in an end volume of $6 \mu \mathrm{L}$, suitable for injection in a mouse knee joint, the MSC secretome was concentrated by loading on a 3 kDa cut-off filter (Amicon Ultra-4 Centrifugal Filter Unit, UFC800324, Merck Millipore B.V.) and spinning down for $20 \mathrm{~min}$ at $4000 \times \mathrm{g}$. Molecules above $3 \mathrm{kDa}$ were retained. The concentrated MSC secretome was collected, aliquoted and stored at $-80{ }^{\circ} \mathrm{C}$ for further use. Concentrated secretome was checked for indoleamine 2,3-dioxygenase (IDO) activity by measuring the metabolite L-kynurenine concentration, as described before (van Buul et al., 2012). Briefly, the concentrated secretome was diluted 10 times and $200 \mu \mathrm{L}$ of the diluted sample were mixed with $30 \%$ trichloroacetic acid (Sigma-Aldrich), incubated at $50{ }^{\circ} \mathrm{C}$ for $30 \mathrm{~min}$ and spun down at $10,000 \times g$ for $5 \mathrm{~min} .75 \mu \mathrm{L}$ of supernatant were added together with $75 \mu \mathrm{L}$ of 7,12-dimethylbenzanthracene (DMBA; Sigma-Aldrich) (20 mg/mL in acetic acid). The extinction was measured at $490 \mathrm{~nm}$ in a Versamax microplate reader (Molecular Devices, LLC, San Jose, CA, USA). A L-kynurenine concentration of $33.7 \pm 4.5 \mu \mathrm{M}$ was calculated considering the dilution factor, indicating substantial immunomodulatory MSC activity. To develop the concentrated secretome production protocol, the secretomes of three MSC donors were used. Increased IDO activity confirmed the anti-inflammatory capacity of the donors. A minimum of $0.05 \%$ of BSA was needed in the secretome to maintain the secreted cytokines and growth factors, based on the concentrations of interleukin 6 (IL-6). The concentration of IL-6 in the concentrated secretome was measured by enzyme-linked immunosorbent assay (ELISA; R\&Dsystems) according to the protocol supplied by the manufacturer. In the obtained batch of secretome, the concentration of IL- 6 was $238 \pm 42 \mathrm{ng} / \mathrm{mL}$ if $0.05 \%$ BSA was present and lower and below the detection limit if no BSA was present (data not shown). Later, this developed protocol was used to produce concentrated secretomes of three new donors, in which the IDO activity was tested to confirm the donors' anti-inflammatory capacity. The MSC secretomes of these donors were pooled and used for in vivo experiments. Control medium was subjected to the same handling as the MSC secretome, including $24 \mathrm{~h}$ incubation and concentration, except for the exposure to the MSCs, and stored at $-80^{\circ} \mathrm{C}$ for further use.

\section{Animal experimental design}

Experiments were performed on 54 male C57/B16 mice, age 12 weeks (Harlan Netherlands B.V., Horst, the Netherlands), with approval of the animal ethical committee (\#EMC 116-14-03). Mice were housed in groups of three or four mice per cage, under $12 \mathrm{~h}$ light-dark cycle at a temperature of $24{ }^{\circ} \mathrm{C}$ and had access to water and food ad libitum at the animal testing facilities of the Erasmus MC, University Medical Centre, Rotterdam, the Netherlands. Before the start of the experiments, mice were allowed to acclimatise for a week. OA was induced unilaterally in the knee of all mice by 2 intra-articular injections of 3 units collagenase type VII (Sigma-Aldrich) at days -7 and -5 . All intra-articular injections were applied with an injection volume of $6 \mu \mathrm{L}$ and under $2.5 \%$ isoflurane anaesthesia, using a $50 \mu \mathrm{L}$ glass syringe (Hamilton Company, Ghiroda, Romania) and a $30 \mathrm{G}$ needle (BD). Contralateral control knees were kept naïve and were not injected. Mice were randomly assigned to either treatment group A, which was injected with 20,000 passage 3 MSCs in $6 \mu \mathrm{L}$ concentrated medium, treatment group B, injected with MSC-secretome from 20,000 MSCs concentrated in $6 \mu \mathrm{L}$ medium or control group C, injected with $6 \mu \mathrm{L}$ control medium $(n=11$ mice per group, 1 mouse in the control group was not assessed by histology due to technical problems). MSCs used in group A were not activated by stimulating medium prior to injection, since in vivo activation by the osteoarthritic environment was considered to be more clinically relevant. All groups received three consecutive injections: the first injection (referred to as day 0 ) was given $7 \mathrm{~d}$ after the first collagenase injection and repeated at day 2 and 4 . Once weekly, weight distribution over the left and right hind limbs was evaluated as an indicator of pain (for the method see below). Animals were euthanised at day 21 after start of treatment and knee joints were harvested for histological analysis. To assess the early effects of the treatments - especially on synovial inflammation an additional experiment was performed consisting of 7 mice in each group (same 3 treatment groups as mentioned previously, $n=7$ mice per group). These animals underwent identical OA induction and treatment protocols. They were euthanised at day 5 after the treatment start and knee joints were prepared for histological evaluation.

\section{Measurement of hind limb weight distribution}

Hind limb weight distribution, as an indicator of pain, was monitored by an Incapacitance Tester (Linton Instrumentation, Norfolk, UK), as previously described (Khatab et al., 2018; van Buul et al., 2014). Mice were positioned on the Incapacitance Tester with each hind limb resting on a separate force plate. Animals were habituated to the apparatus, starting 2 weeks prior to the experiments. The examiner performing the measurements was blinded to the data registration. Measurements were automatically stored in a computer database. 
A baseline measurement was performed at day -7 , just before the induction of the OA. Follow up measurements were performed 1 week after OA induction at day 0 , just before therapy administration and at day 7, 14 and 21. After the 3 weeks of data collection, upon data analyses, measurements with a registration below $3 \mathrm{~g}(<10 \%$ of total body weight) per hind limb or less than $10 \mathrm{~g}(<30 \%$ of total body weight) in total over both hind limbs were excluded. 30-45 measurements were recorded per time point, 15 measurements per time point per animal were available on average. For each time point per mouse, the average of these measurements was used to calculate the percentage of weight on the affected limb as an indication of pain in the affected limb. The average value per measurement time point was used for statistical analyses.

\section{Histology}

Knees were fixed in $4 \%$ formalin (v/v) for 1 week, decalcified in $10 \%$ EDTA for 2 weeks and embedded in paraffin. Coronal sections of $6 \mu \mathrm{m}$ were cut for analysis of synovial inflammation and cartilage damage.

\section{Cartilage damage}

Cartilage damage was evaluated on thionine-stained sections by two observers blinded to the treatment groups using the scoring system described by Glasson et al. (2010). Briefly, this score ranges from 0 , for normal cartilage, to 6 , for cartilage with clefts and erosion to the calcified cartilage in $>75 \%$ of the articular surface. For each knee, cartilage quality in the lateral and medial compartment - both femur and tibia - of the knee was scored on 3 sections at standardised locations in the knee with $180 \mu \mathrm{m}$ between the sections, leading to a maximum score of 12 per compartment and a total summed score of 36 per compartment. For each compartment, the maximum and summed score assigned by the two blinded observers was averaged. Since OA damage was most pronounced in the lateral compartment (data not shown), these data were considered for further analyses.

\section{Subchondral bone changes}

The percentage of subchondral bone per total volume of subchondral space between calcified cartilage and growth plate was calculated. Measurements were performed using ImageJ software $(\mathrm{NIH})$. For each knee, a region of interest was drawn in a standardised way on 3 sections at standardised locations in the knee, with $180 \mu \mathrm{m}$ between the sections. The region of interest was drawn between the calcified cartilage on the top and the growth plate on the bottom. The insertion of the cruciate ligaments was used as medial boundary and the lateral side of the growth plate as lateral boundary, taking care not to include osteophytes. Since the largest amount of remodelling was found in the medial compartment (data not shown), these data were considered for further analyses.

\section{Synovial inflammation}

Sections were stained with haematoxylin and eosin for synovial inflammation assessment. Images were acquired using the NanoZoomer Digital Pathology program (Hamamatsu Photonics, Ammersee, Germany). Synovial thickness was measured from the capsule to the superficial layer of the synovial membrane at the medial and lateral sides of the parapatellar recesses (three positions per section), as previously described (Khatab et al., 2018). These measurements were performed on three sections per knee, with $180 \mu \mathrm{m}$ between the sections. The thickness measurements were averaged to obtain a single value per knee joint.

To evaluate macrophage subtypes in the synovial membrane, inducible nitric oxide (iNOS) was used as a marker of pro-inflammatory macrophages, CD163 as a marker of anti-inflammatory macrophages and CD206 as a marker of tissue repair macrophages (Park et al., 2017; Utomo et al., 2016). For this purpose, sections were deparaffinised, washed and heat-mediated antigen retrieval was performed for CD163 and CD206, by placing the slides in $95^{\circ} \mathrm{C}$ citrate buffer $(\mathrm{pH} \mathrm{6)}$ for $20 \mathrm{~min}$. Antigen retrieval for iNOS was performed by placing the slides in $95^{\circ} \mathrm{C}$ Tris-EDTA buffer ( $\mathrm{pH}$ 9) for $20 \mathrm{~min}$. Blocking of specific binding was performed with $10 \%$ goat serum (Southern Biotech, Birmingham, AL, USA) for $30 \mathrm{~min}$. Hereafter, sections were incubated for $1 \mathrm{~h}$ with the primary antibodies iNOS $(2.0 \mu \mathrm{g} / \mathrm{mL}, \# 15323$,

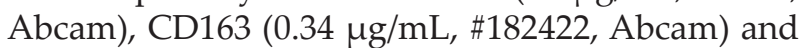
CD206 (2.5 $\mu \mathrm{g} / \mathrm{mL}$, \#64693, Abcam), followed by $30 \mathrm{~min}$ incubation with a biotinylated anti-rabbit Ig link (HK-326-UR, Biogenex, Fremont, CA, USA), diluted $1: 50$ in PBS/1 \% BSA. Thereafter, sections were incubated with an alkaline-phosphataseconjugated streptavidin label (HK-321-UK, Biogenex) diluted $1: 50$ in PBS/1 \% BSA. To reduce background, endogenous alkaline phosphatase activity was inhibited with levamisole (Sigma-Aldrich Chemie). New Fuchsin (Fisher Scientific) and Napthol AS-MX phosphate (Sigma-Aldrich Chemie) substrates were used for colour development and counterstaining was performed with haematoxylin. As a negative control, rabbit IgG antibody (DakoCytomation, Glostrup, Denmark) was used. The sections were ranked from the weakest to strongest staining for iNOS, CD163 and CD206 by two observers blinded to the treatment groups. When multiple sections had similar staining strength, i.e. amount of positive cells, the rank mean was assigned to each section. The average of the ranks assigned by the two observers to the knee for a certain staining was used for further analyses.

\section{Statistical analysis}

Data were analysed with IBM SPSS statistics 24 (SPSS, Chicago, IL, USA). For the effect on weight 
distribution, normality per measurement time point was confirmed with a Shapiro Wilk test. For pain measurements, the collagenase effect was analysed using a two-tailed paired $t$-test before and after OA induction. For treatment effects, all groups were compared using a linear mixed model in which measurement time point and treatment were considered fixed factors and weight-bearing a dependent factor. Post-hoc analyses were performed by Bonferroni correction. Quantitative histology data were analysed by means of a univariate general linear model where measurement time point and treatment were considered fixed factors and subchondral bone volume percentage a dependent variable. Semiquantitative histology scores were compared using a non-parametric Mann-Whitney test, to assess collagenase or measurement time point effects, and Kruskal Wallis test, to assess treatment effects within different time points. Binominal histology data were analysed by means of $\chi^{2}$ test for multiple group testing and Fisher's exact test for comparing separate groups. For all tests, $p<0.05$ was considered statistically significant. Correlation analysis was performed by means of a Spearman's Q test. For the interpretation of the correlation coefficient, the absolute value of $r_{\mathrm{s}}$ was used, classifying the correlations as weak $(<0.39)$, moderate (0.40-0.59), strong (0.60-0.79) and very strong $(>0.80)$. Correlations were regarded significant if $p<0.05$.

\section{Results}

Injection with MSC secretome resulted in early pain reduction

Weight distribution over the hind limbs was determined as an indicator of pain (Fig. 1). On day -7 , just before induction of $\mathrm{OA}$, the average weight on the right hind limb for all groups was $49.9 \pm 1.7 \%$, indicating no difference between left and right hind limbs. $7 \mathrm{~d}$ after induction of OA (day 0, before treatment), $41.2 \pm 6.3 \%$ of the weight was distributed on the OA-affected limb, indicating pain when compared to before OA induction (Fig. 1, $p<0.001$ ). No differences between treatment groups were found before both OA induction and treatment. At day 7, significantly more weight was put on the OA-affected limb by MSC-secretome- $(49.5 \pm 2.2 \%)$ and MSC$(47.7 \pm 2.9 \%)$ injected animals at day 7 , compared to control animals $(43.6 \pm 4.2 \% ; p=0.001$ and $p=0.023$ respectively). Furthermore, the MSC-secretome- and MSC-injected animals put significantly more weight on their OA-affected limb on day 7 as compared to day $0(p=0.013$ and $p=0.032$ respectively, not shown in graph) whereas control animals did not, indicating early normalisation in weight bearing and pain reduction in treated animals. At day 14 and day 21, all groups showed less pain when compared to day 0 , whereas no significant difference between groups was observed.
MSC secretome had a protective effect on cartilage damage but not on subchondral bone remodelling To evaluate cartilage damage, all knees were scored $21 \mathrm{~d}$ after treatment (Fig. 2a,b, score range 0-12 for max score and 0-36 for sum score). Due to a high variability in the development of OA, no significant differences were found by non-parametric tests when comparing OA scores between knees treated with collagenase and healthy knees or among any of the treatment groups (Fig. 2a,b). Since some animals showed a clear OA development whereas others did not, binominal data were generated, where a max OA score of $>2$ was considered as having OA and a score of $\leq 2$ as not having OA (Table 1). A significant difference when comparing all groups was found using $\chi^{2}$ test $(p=0.044)$, after which separate groups were tested using Fisher's exact test. In the control group, where collagenase was injected but no MSC treatment was applied, more knees had OA than in the healthy group $(p=0.012)$. OA development was prevented in knees that were treated with MSC secretome or MSCs, in which no significant difference was found between healthy knees ( $p=0.238$ for both). No significant effect was observed when directly comparing MSC-secretome- or MSC-injected knees to control knees ( $p=0.183$ for both).

Identical analyses were performed for the sum OA scores (Fig. 2b), where a sum OA score of $>3$ was considered as having $\mathrm{OA}$ and a sum score of $\leq 3$ as not having OA (data not shown). Although the control group contained most animals with OA, no significant difference among all groups regarding sum OA scores was found by $\chi^{2}$ test; consequently, no further analyses were performed to evaluate treatment effects.

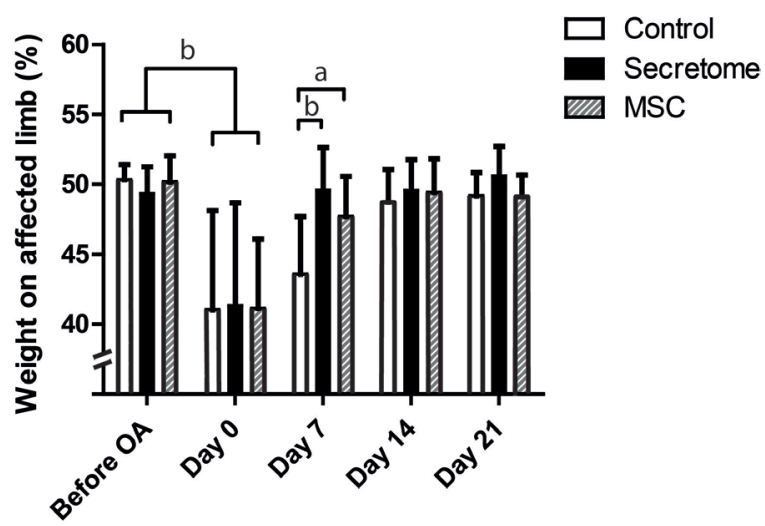

Fig. 1. Assessment of pain with hind limb weight distribution. Hind limb weight distribution was measured as an index of joint pain. OA induction by collagenase caused significant pain. MSC secretome and MSC injection resulted in normalisation of weight bearing at day 7 , at which time point pain was significantly reduced when compared to the control group and to day 0 . At day 14 and 21, all groups showed significantly less pain as compared to day 0 . Data shown as mean $\pm \mathrm{SD}, n=11$ /group, ${ }^{\mathrm{a}} p<0.05 ;{ }^{\mathrm{b}} p<0.001$. 
To further appraise the influence of MSC secretome on OA processes, the effects on subchondral bone remodelling at day 5 and day 21 after treatment were determined (Fig. 3a,b). Although subchondral bone volume appeared to be slightly decreased in knees with induced OA, no significant difference between any of the groups was found.

\section{MSC secretome had no effect on synovial membrane inflammation}

All collagenase-injected knees displayed a significantly thickened synovial membrane when compared to healthy knees at 5 and $21 \mathrm{~d}$ after treatment (Fig. 4a, b; $p<0.001$ for both time points). No significant difference was observed between treatment groups within each time point. Overall, synovial membrane thickness was largely reduced over time between day 5 and day 21 for all groups $(p<0.001)$.

To further analyse the synovial inflammation processes, the presence of different macrophage subtypes was assessed by the presence of iNOS $^{+}$ (pro-inflammatory), CD163 ${ }^{+}$(anti-inflammatory) and $\mathrm{CD}^{2} \mathrm{6}^{+}$(tissue repair) cells in the different treatment groups. The presence of iNOS ${ }^{+}$cells was not significantly different between the treatment groups at each time points individually or between both time points (Fig. 4c). A trend was observed towards a lower presence of iNOS $^{+}$cells in the control vs. MSC group at day $5(p=0.053)$. A significant increase in the presence of $\mathrm{CD}_{163}{ }^{+}$and CD206 ${ }^{+}$cells was found in all groups at day 21 vs. day $5(p<0.001$ and $p=0.004$, respectively), but no differences in the presence of $\mathrm{CD} 163^{+}$or $\mathrm{CD} 206^{+}$cells were observed between treatment groups at both time points.

To further unravel the inflammatory processes and their relation to pain and structural damage, these aspects were correlated to the presence of the different macrophage subtypes (Table 2). An increased staining for $\mathrm{CD}_{163}{ }^{+}$cells (anti-inflammatory macrophages) was moderately associated with a thinner synovial membrane [correlation coefficient $(C C)=-0.397$, $p=0.003$, all treatment groups combined], but not with cartilage damage. This could indicate that more staining for anti-inflammatory macrophages was related to less synovial inflammation. In addition, the amount of $\mathrm{CD}_{163^{+}}$staining was moderately associated with less pain at day $14(C C=-0.394$; $p=0.026$ ), possibly pointing towards pain reduction in the presence of anti-inflammatory macrophages. $\mathrm{CD}_{163}{ }^{+}$staining was weakly associated with the intensity of $\mathrm{CD}^{2} 6^{+}$staining $(\mathrm{CC}=0.281 ; p=0.042)$, representing the co-presence of anti-inflammatory and repair-related macrophages. CD206 ${ }^{+}$staining was moderately associated with both lateral $(C C=0.465$; $p=0.007)$ and medial OA damage $(C C=0.412$; $p=0.028)$, but not with synovial thickness. This could indicate a higher presence of tissue-repair-associated macrophages in the situation of more cartilage damage. Lastly, a moderate association between subchondral bone volume and synovial thickness was found $(C C=0.496, p<0.001)$, possibly indicating an increased bone remodelling with increased synovial inflammation. No significant associations were found between NOS $^{+}$staining and pain, synovial thickness or cartilage damage. There were no associations between pain and synovial thickness, OA score or subchondral bone volume.

\section{Discussion}

Injection of human MSCs secretome resulted in an early pain reduction and had a protective effect on the development of cartilage damage in a mouse collagenase-induced OA (CIOA) model. No clear treatment effects were observed on subchondral
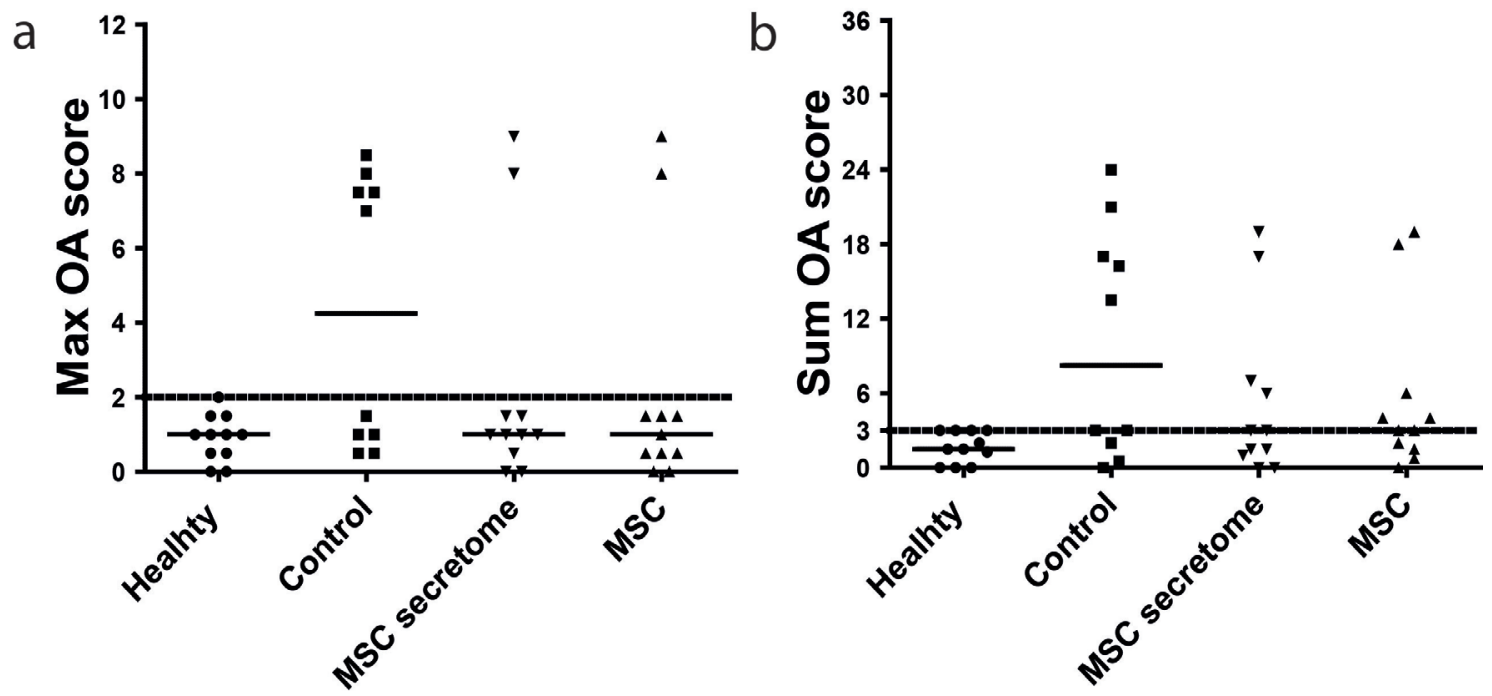

Fig. 2. Maximum and sum OA scores for the lateral compartment were not significantly different between any of the groups $(\mathbf{a}, \mathbf{b})$. Individual values are shown, solid lines represent median values, dotted line represents cut-off value for definition of OA development or not. 
Table 1. OA incidence in healthy and treated knees. A maximum OA score of 2 was used as cut-off. A significant difference was found when comparing the binominal data of OA incidence in control versus healthy knees. No significant difference was found between MSC-secretome or MSC-treated knees as compared to healthy knees, indicating a chondroprotective effect. ${ }^{a} p<0.05$.

\begin{tabular}{|c|c|c|c|c|}
\hline OA incidence & Healthy & Control & MSC secretome & MSC \\
\hline $\mathrm{AO}$ & 0 & 5 & 2 & 2 \\
\hline Non-OA & 11 & 5 & 9 & 9 \\
\hline Percentage $\mathrm{AO}$ & $0.0 \%$ & $50.0 \%$ & $18.2 \%$ & $18.2 \%$ \\
\hline
\end{tabular}

bone remodelling or synovial inflammation, although several significant moderate correlations between macrophage phenotypes and OA characteristics were found. These correlations enhanced the understanding of the inflammation role in the mouse CIOA model. MSC secretome injections were at least as effective as MSCs in the amelioration of OArelated pain or morphological changes, endorsing the potential of this cell-free approach.

MSCs diminish several OA characteristics, as demonstrated in pre-clinical and some initial clinical studies (Gupta et al., 2016; Lamo-Espinosa et al., 2016; Murphy et al., 2003; Pers et al., 2016; Ter Huurne et al., 2012; van Buul et al., 2014). Although intra-articular injection of MSCs does benefit the joint after OA induction, the MSCs themselves are not detectable 3 weeks after injection (van Buul et al., 2014). This limited presence of the MSCs in the joint is also observed by Diekman et al. (2013) and Mak et al. (2016), the latter suggesting that this is due to differentiation, migration or cell death. Thus, although long-term engraftment is not present, intraarticular injection of MSC can have a beneficial effect, leading Prockop (2009) and von Bahr et al. (2012) to postulate the 'hit-and-run' mechanism, in which the MSC-secreted factors play an important role. The use of the therapeutic MSC secretome without actual employment of cells originates from the cardiovascular field (Gnecchi et al., 2005). Although the longevity of the secreted factors is expected to be even lower than that of the MSCs, they seem to have an effect in vivo, possibly by activating a cascade of reactions.

It is not known which factor(s) in the MSCsecretome is responsible for the anti-osteoarthritic effect. Next to IL-6, prostaglandin E2 (PGE2), TNFstimulated gene 6 (TSG-6) and hepatocyte growth factor (HGF), which are partly responsible for some effects in various disease models (reviewed by Madrigal et al., 2014), extracellular vesicles secreted by the MSCs are speculated to be important for injury reduction and repair in, for example, experimental myocardial infarction, stroke or endotoxin-induced lung injury, as reviewed by Konala et al. (2016) and Rani et al. (2015). In the orthopaedic field, Platas et al. (2013) demonstrate protective effects of adiposetissue-derived MSC secretome in an inflammatory in vitro chondrocyte model. Other groups show beneficial effects of MSC-derived exosomes and/or particles in various pre-clinical OA models in vivo
(Cosenza et al., 2017; Tao et al., 2017; Wang et al., 2017; Zhang et al., 2018; Zhu et al., 2017), as reviewed by Toh et al. (2016). Although the extracellular vesicle content in the MSC secretome was not quantified, a large proportion of the extracellular vesicles, mainly the exosomes (10-100 nm) and microvesicles (100$1000 \mathrm{~nm}$ ), was most likely retained (Morhayim et al., 2014). The retention of the extracellular vesicles is described by the manufacturer when using the Amicon Ultra-4 Centrifugal Filter Unit, even with a larger $10 \mathrm{kDa}$ cut-off. Therefore, the observed effects were due, possibly, to the MSC extracellular vesciles, which provide a controlled microenvironment and protect their content from degradation in vivo thanks to their bilayered membrane (Zheng et al., 2018). These in vivo effects were promising and encourage the further unravelling of the MSC therapeutic function. Nevertheless, these cited studies are based on extracellular vesicles isolated from the secretome of young animals, human embryonic stem cells or genetically modified cells. To our knowledge, this was the first evidence of in vivo anti-osteoarthritic effects of MSC secretome isolated from aged human donors with end-stage OA.

Although promising, the MSC secretome could also contain components causing an adverse effect. Additional proteomic studies are needed to examine the exact components of the secretome, to further elucidate its working mechanism. The secretome use would greatly reduce problems linked to safety and legal issues related to cell-based therapies, thereby emphasising the clinical relevance of these findings. If the MSC secretome could be used in OA therapies as an alternative to MSCs, the possibilities to provide a more standardised and affordable therapeutic option would be largely increased. Good manufacturing practice (GMP) facilities could become more centralised and large quantities of MSC secretome could be generated from well-defined, selected batches of MSCs. The secretome could be checked for predetermined concentrations of cytokines and growth factors. These could either be preferable or non-preferable cytokines depending on the application. Simultaneously, problems raised by legal and safety issues regarding the use of cell therapy would be decreased. Nevertheless, such an approach might bring new safety and regulatory issues, such as finding suitable MSCs donors for maintaining a large scale secretome production in vitro. 

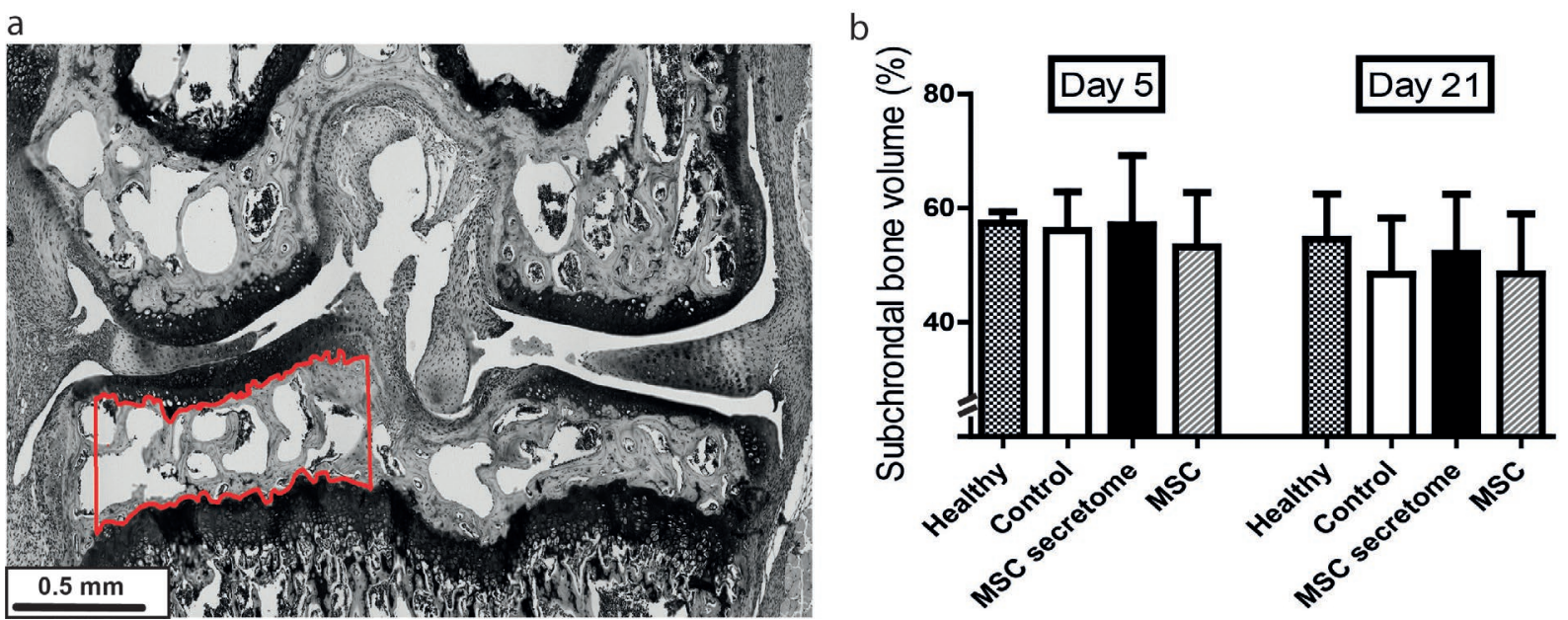

Fig. 3. No treatment effect on subchondral bone volume. $(\mathbf{a}, \mathbf{b})$ The subchondral bone volume was calculated as a percentage of total volume of subchondral space between calcified cartilage and growth plate, depicted in a. No differences were found between healthy and OA-induced knees or any of the treatment groups. Data shown as mean \pm SD for medial tibial plateau, $n=7 /$ group at day 5 and $n=11$ at day 21; healthy knees $n=5$ at day 5 and $n=6$ at day 21 .

a

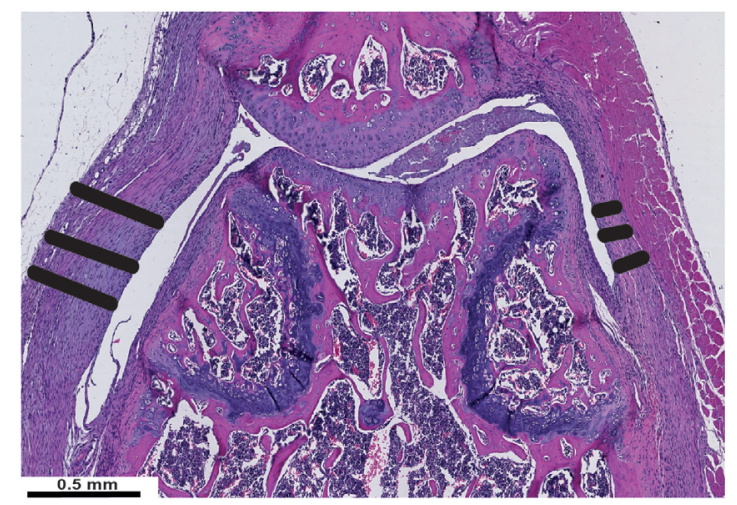

b

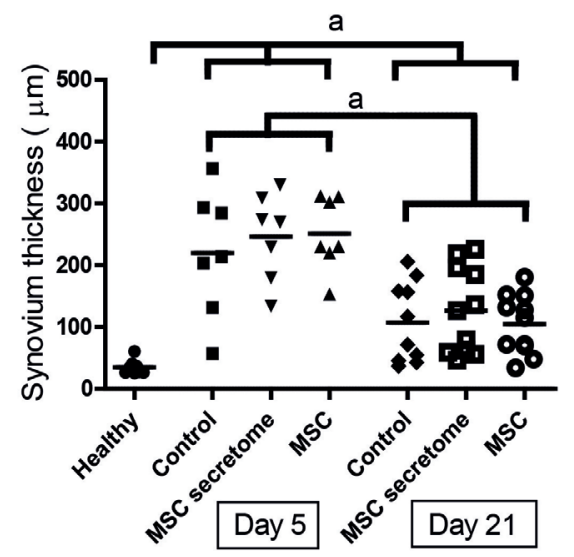

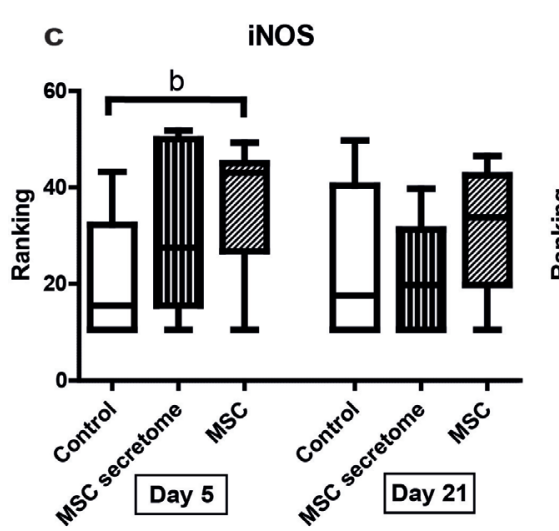
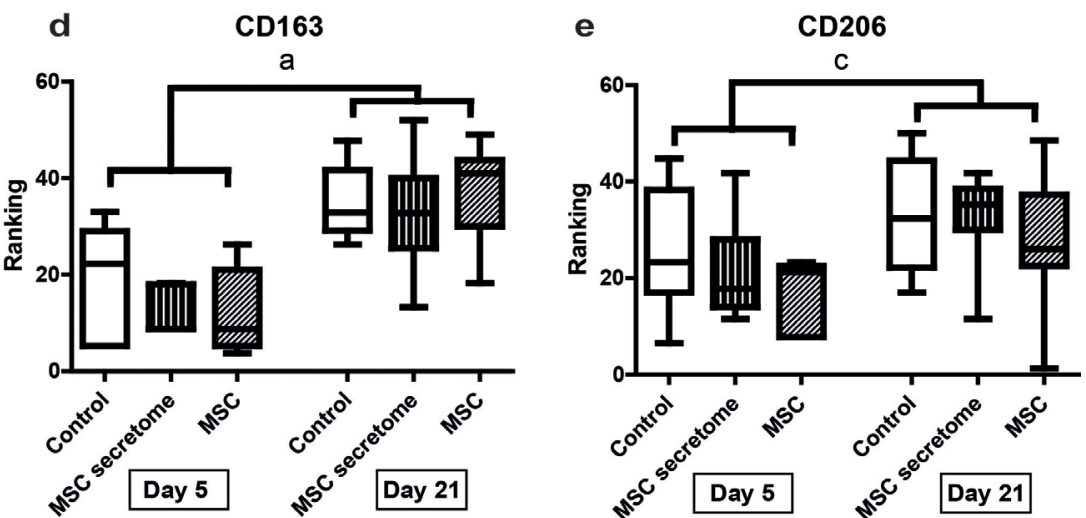

Fig. 4. No treatment effect on synovial membrane thickness (a,b) Synovial membrane thickness decreased in time. Individual values are shown, lines represent mean values. (c) A trend towards more iNOS ${ }^{+}$staining was observed in the MSC group at day 5. The amount of (d) $\mathrm{CD}_{163}{ }^{+}$and (e) CD206 ${ }^{+}$staining increased with time. Box and whiskers plots are shown, lines represent median values. $) .{ }^{a} p<0.001 ;{ }^{b} p=0.053 ;{ }^{c} p<0.005$ 
Table 2. Selection of correlations coefficients between the parameters tested. Associations between $\mathrm{CD} 163^{+}$ and $\mathrm{CD}_{206}{ }^{+}$staining and various OA aspects as reported in previous figures. ${ }^{a}$ Weight distribution on the affected limb was measured as an indicator of pain; the higher the percentage of weight on the affected limb the less pain, thus giving a negative association with $\mathrm{CD}_{163}{ }^{+}$cells. Synovial thickness, subchondral bone volume and macrophage data based on day 5 and day 21 time points, OA score based on day 21 time point only.

\begin{tabular}{|c|c|c|c|c|c|}
\hline \multicolumn{2}{|c|}{ Parameters tested by Spearman o } & \multirow{2}{*}{$\begin{array}{c}\begin{array}{c}\text { Correlation } \\
\text { coefficient }\end{array} \\
-0.397\end{array}$} & \multirow{2}{*}{$\begin{array}{l}\text { Strength } \\
\text { Moderate }\end{array}$} & \multirow{2}{*}{$\underline{p \text {-value }}$} & \multirow{2}{*}{\begin{tabular}{|c|} 
Interpretation \\
$\begin{array}{c}\text { Higher CD163 staining intensity } \\
\text { associated with thinner synovial } \\
\text { membrane }\end{array}$ \\
\end{tabular}} \\
\hline CD163 + cells & $\begin{array}{c}\text { Synovial membrane } \\
\text { thickness }\end{array}$ & & & & \\
\hline CD163 + cells & Pain day 14 & -0.394 & Moderate & 0.026 & $\begin{array}{c}\text { Higher CD163 staining intensity } \\
\text { associated with less pain at day } \\
14^{\mathrm{a}} \\
\end{array}$ \\
\hline CD163 + cells & $\mathrm{CD}^{206^{+}}$cells & 0.281 & Weak & 0.042 & $\begin{array}{c}\text { Higher CD163 staining intensity } \\
\text { associated with more repair } \\
\text { macrophages }\end{array}$ \\
\hline CD206 + cells & Lateral OA score & 0.465 & Moderate & 0.007 & $\begin{array}{c}\text { Higher CD206 staining intensity } \\
\text { associated with more lateral OA } \\
\text { damage }\end{array}$ \\
\hline CD206 + cells & Medial OA score & 0.412 & Moderate & 0.019 & $\begin{array}{c}\text { Higher CD206 staining intensity } \\
\text { associated with more medial OA } \\
\text { damage }\end{array}$ \\
\hline $\begin{array}{l}\text { Subchondral } \\
\text { bone volume }\end{array}$ & $\begin{array}{c}\text { Synovial membrane } \\
\text { thickness }\end{array}$ & 0.496 & Moderate & $<0.001$ & $\begin{array}{c}\text { Larger subchondal bone volume } \\
\text { associated with thicker synovial } \\
\text { membrane }\end{array}$ \\
\hline
\end{tabular}

The pain reduction by treatment with MSC secretome or MSCs, as seen in the present study, lasted for the entire experiment. Interestingly, the control group showed a pain reduction as well, only at a later time point. This indicated a general pain reduction as the natural course of the used OA model, as found before (Khatab et al., 2018). This is also described by Adaes et al. (2014), who find a maximum of pain sensation 1 week after $O A$ induction by collagenase in a rat model. This pain sensation gradually decreased in a period of 4 to 6 weeks after induction. Clinically, OA-related pain is correlated to the radiological presence of synovial inflammation as well as bone marrow lesions, as seen by magnetic resonance imaging (Carotti et al., 2017). In the CIOA model, both synovial inflammation and dynamic subchondral bone changes are present (Botter et al., 2011; van der Kraan et al., 1990). Osteochondral angiogenesis is shown in OA, in which neural growth factor expression and sensory nerve growth may be the links to perceived pain (Mapp and Walsh, 2012). Since cartilage itself is an aneural tissue, this is a plausible mechanistic explanation for pain perception in OA. Subchondral bone changes are biphasic during OA: an initial atrophic phase leads to a decreased subchondral bone volume, after which a hypertrophic phase initiates the increase in bone volume (reviewed by Yuan et al., 2014). Botter et al. (2011) find initial thinning of the subchondral bone plate 2 weeks after OA induction in a mouse CIOA model, after which, at 10 weeks, subchondral bone thickness returns to levels comparable to before OA induction. Clinically, fully eroded joint regions display a thickened subchondral bone region, whereas partially eroded areas show a thinner subchondral bone plate as compared to non-eroded regions (Hwang et al., 2008). Regarding the effect of mesenchymal cells on subchondral bone, Parrilli et al. (2017) show that injection of adipose-derived stromal cells counteracted accelerated bone turnover in a rabbit OA model. A trend towards a decrease in bone volume was observed in the used CIOA model, but no effects of the different treatments nor a correlation between pain reduction and subchondral bone volume were found. The discrepancy in the absence of an MSC effect in the present study could possibly be due to the fact that subchondral bone volume was calculated on histological slides instead of on true 3D volumetric or densitometric bone measurements by micro-computed tomography $(\mu \mathrm{CT})$.

The first phase after collagenase injection in the CIOA model is an inflammatory phase, leading to synovial thickening and infiltration with inflammatory cells within 1 week. Cartilage damage and other pathological OA-related changes become more pronounced after 2 to 4 weeks, with marked OA changes found after 6 weeks (Adaes et al., 2014; van der Kraan et al., 1990). Although a clear pain reduction in MSC-secretome- and MSC-treated animals was observed 1 week after treatment - which is 2 weeks after OA induction - no treatment effects on synovial thickness or synovial macrophage phenotype were 
found at that time point nor at a later stage. In fact, a trend towards a larger presence of iNOS ${ }^{+}$cells was observed in MSC-treated knees, which could indicate a more pro-inflammatory phenotype. Synovial inflammation most likely played a role in pain perception, partly given the fact that pain and synovial inflammation both diminished in time. Nevertheless, it was not possible to relate the early analgesic effect of MSC secretome or MSCs to this OA characteristic. Ter Huurne et al. (2012) observe a clear anti-inflammatory effect after injection of adipose-derived stem cells in a mouse collagenase model. The discrepancy between this report and the present study's findings could possibly be explained by the use, in the present study, of human cells in an immunocompetent mice strain. Other groups describe that although immuno-privileged, MSCs maintain a degree of immunogenicity (Schu et al., 2012). A trend towards increased synovial inflammation is observed after injection of allogeneic rat MSCs in a rat mono-iodoacetate (MIA) model of OA (van Buul et al., 2014). This increased synovial inflammation is significant when using xenogeneic human MSCs (van Buul et al., 2014), further pointing towards the maintenance of the MSC immunogenicity. In this aspect, the use of the MSC secretome is likely to be safer, because the concentration of immune complexes - possibly present on the extracellular vesicles - in the secretome is lower than with cells, causing a weaker host inflammatory response (Rani et al., 2015). Although there is the possibility that some factors in the human MSC secretome might not crossreact with the mouse immune system. Therefore, it is not possible to conclude that the secretome showed its full potential. Furthermore, the secretome could contain components causing an adverse effect. Additional proteomic studies are needed to examine the exact components of the secretome, to further elucidate its working mechanism.

In conclusion, human MSC secretome from patients with end-stage OA was shown to diminish pain and structural OA-related changes in a mouse CIOA model. These effects were at least as effective as injection of MSCs themselves. The use of MSCsecreted factors instead of the cells themselves provides options to enhance standardisation, affordability and efficacy of this therapeutic approach. The study's results encourage further development of this strategy towards cell-based treatments as a true disease-modifying anti-osteoarthritic drug with wide clinical availability.

\section{Acknowledgements}

This work was financially supported by grants from the Netherlands Organisation for Scientific Research (Aspasia), Applied and Engineering Sciences (AES, previously Technology Foundation STW) and Austrian Research Promotion Agency (FFG Bridge Grant, Fetocart). The authors would like to thank
Erwin J.H. Waarsing for his help with the statistical analyses.

\section{References}

Adaes S, Mendonca M, Santos TN, Castro-Lopes JM, Ferreira-Gomes J, Neto FL (2014) Intra-articular injection of collagenase in the knee of rats as an alternative model to study nociception associated with osteoarthritis. Arthritis Res Ther 16: R10.

Berenbaum F (2013) Osteoarthritis as an inflammatory disease (osteoarthritis is not osteoarthrosis!). Osteoarthritis Cartilage 21: 16-21.

Barrachina L, Remacha AR, Romero A, Vazquez FJ, Albareda J, Prades M, Gosalvez J, Roy R, Zaragoza P, Martin-Burriel I, Rodellar C (2017) Priming equine bone marrow-derived mesenchymal stem cells with proinflammatory cytokines: implications in immunomodulation-immunogenicity balance, cell viability, and differentiation potential. Stem Cells Dev 26: 15-24.

Botter SM, van Osch GJ, Clockaerts S, Waarsing JH, Weinans H, van Leeuwen JP (2011) Osteoarthritis induction leads to early and temporal subchondral plate porosity in the tibial plateau of mice: an in vivo microfocal computed tomography study. Arthritis Rheum 63: 2690-2699.

Caplan AI (2017) Mesenchymal stem cells: time to change the name! Stem Cells Transl Med 6: 1445-1451.

Caplan AI, Dennis JE (2006) Mesenchymal stem cells as trophic mediators. J Cell Biochem 98: 10761084.

Carotti M, Salaffi F, Di Carlo M, Giovagnoni A (2017) Relationship between magnetic resonance imaging findings, radiological grading, psychological distress and pain in patients with symptomatic knee osteoarthritis. Radiol Med 122: 934-943.

Cosenza S, Ruiz M, Toupet K, Jorgensen C, Noel D (2017) Mesenchymal stem cells derived exosomes and microparticles protect cartilage and bone from degradation in osteoarthritis. Sci Rep 7: 16214.

Crop MJ, Baan CC, Korevaar SS, Ijzermans JN, Pescatori M, Stubbs AP, van Ijcken WF, Dahlke MH, Eggenhofer E, Weimar W, Hoogduijn MJ (2010) Inflammatory conditions affect gene expression and function of human adipose tissue-derived mesenchymal stem cells. Clin Exp Immunol 162: 474-486.

de Mos M, Koevoet WJ, Jahr H, Verstegen MM, Heijboer MP, Kops N, van Leeuwen JP, Weinans H, Verhaar JA, van Osch GJ (2007) Intrinsic differentiation potential of adolescent human tendon tissue: an in-vitro cell differentiation study. BMC Musculoskelet Disord 8: 16.

Diekman BO, Wu CL, Louer CR, Furman BD, Huebner JL, Kraus VB, Olson SA, Guilak F (2013) Intra-articular delivery of purified mesenchymal stem cells from C57BL/6 or MRL/MpJ superhealer mice prevents posttraumatic arthritis. Cell Transplant 22: 1395-1408. 
Eggenhofer E, Luk F, Dahlke MH, Hoogduijn M] (2014) The life and fate of mesenchymal stem cells. Front Immunol 5: 148.

Estrada R, Li N, Sarojini H, An J, Lee MJ, Wang E (2009) Secretome from mesenchymal stem cells induces angiogenesis via Cyr61. J Cell Physiol 219: 563-571.

Glasson SS, Chambers MG, Van Den Berg WB, Little CB (2010) The OARSI histopathology initiative - recommendations for histological assessments of osteoarthritis in the mouse. Osteoarthritis Cartilage 18 Suppl 3: S17-23.

Gnecchi M, He H, Liang OD, Melo LG, Morello F, Mu H, Noiseux N, Zhang L, Pratt RE, Ingwall JS, Dzau VJ (2005) Paracrine action accounts for marked protection of ischemic heart by Akt-modified mesenchymal stem cells. Nat Med 11: 367-368.

Gupta PK, Chullikana A, Rengasamy M, Shetty N, Pandey V, Agarwal V, Wagh SY, Vellotare PK, Damodaran D, Viswanathan P, Thej C, Balasubramanian S, Majumdar AS (2016) Efficacy and safety of adult human bone marrow-derived, cultured, pooled, allogeneic mesenchymal stromal cells (Stempeucel(R)): preclinical and clinical trial in osteoarthritis of the knee joint. Arthritis Res Ther 18: 301.

Hwang J, Bae WC, Shieu W, Lewis CW, Bugbee WD, Sah RL (2008) Increased hydraulic conductance of human articular cartilage and subchondral bone plate with progression of osteoarthritis. Arthritis Rheum 58: 3831-3842.

Jo CH, Lee YG, Shin WH, Kim H, Chai JW, Jeong EC, Kim JE, Shim H, Shin JS, Shin IS, Ra JC, Oh S, Yoon KS (2014) Intra-articular injection of mesenchymal stem cells for the treatment of osteoarthritis of the knee: a proof-of-concept clinical trial. Stem Cells 32: 1254-1266.

Khatab S, van Buul GM, Kops N, BastiaansenJenniskens YM, Bos PK, Verhaar JA, van Osch GJ (2018) Intra-articular injections of platelet-rich plasma releasate reduce pain and synovial inflammation in a mouse model of osteoarthritis. Am J Sports Med 46: 977-986.

Kinnaird T, Stabile E, Burnett MS, Shou M, Lee CW, Barr S, Fuchs S, Epstein SE (2004) Local delivery of marrow-derived stromal cells augments collateral perfusion through paracrine mechanisms. Circulation 109: 1543-1549.

Konala VB, Mamidi MK, Bhonde R, Das AK, Pochampally R, Pal R (2016) The current landscape of the mesenchymal stromal cell secretome: a new paradigm for cell-free regeneration. Cytotherapy 18: 13-24.

Lamo-Espinosa JM, Mora G, Blanco JF, GraneroMolto F, Nunez-Cordoba JM, Sanchez-Echenique C, Bondia JM, Aquerreta JD, Andreu EJ, Ornilla E, Villaron EM, Valenti-Azcarate A, Sanchez-Guijo F, Del Canizo MC, Valenti-Nin JR, Prosper F (2016) Intra-articular injection of two different doses of autologous bone marrow mesenchymal stem cells versus hyaluronic acid in the treatment of knee osteoarthritis: multicenter randomized controlled clinical trial (phase I/II). J Transl Med 14: 246.

Le Blanc K, Mougiakakos D (2012) Multipotent mesenchymal stromal cells and the innate immune system. Nat Rev Immunol 12: 383-396.

Madrigal M, Rao KS, Riordan NH (2014) A review of therapeutic effects of mesenchymal stem cell secretions and induction of secretory modification by different culture methods. J Transl Med 12: 260.

Mak J, Jablonski CL, Leonard CA, Dunn JF, Raharjo E, Matyas JR, Biernaskie J, Krawetz RJ (2016) Intra-articular injection of synovial mesenchymal stem cells improves cartilage repair in a mouse injury model. Sci Rep 6: 23076.

Mapp PI, Walsh DA (2012) Mechanisms and targets of angiogenesis and nerve growth in osteoarthritis. Nat Rev Rheumatol 8: 390-398.

Morhayim J, Baroncelli M, van Leeuwen JP (2014) Extracellular vesicles: specialized bone messengers. Arch Biochem Biophys 561: 38-45.

Murphy JM, Fink DJ, Hunziker EB, Barry FP (2003) Stem cell therapy in a caprine model of osteoarthritis. Arthritis Rheum 48: 3464-3474.

Park HS, Nelson DE, Taylor ZE, Hayes JB, Cunningham KD, Arivett BA, Ghosh R, Wolf LC, Taylor KM, Farone MB, Handy ST, Farone AL (2017) Suppression of LPS-induced NF-kappaB activity in macrophages by the synthetic aurone, (Z)-2-((5(hydroxymethyl) furan-2-yl) methylene) benzofuran3(2H)-one. Int Immunopharmacol 43: 116-128.

Parrilli A, Giavaresi G, Ferrari A, Salamanna F, Desando G, Grigolo B, Martini L, Fini M (2017) Subchondral bone response to injected adiposederived stromal cells for treating osteoarthritis using an experimental rabbit model. Biotech Histochem 92: 201-211.

Pers YM, Rackwitz L, Ferreira R, Pullig O, Delfour C, Barry F, Sensebe L, Casteilla L, Fleury S, Bourin P, Noël D, Canovas F, Cyteval C, Lisignoli G, Schrauth J, Haddad D, Domergue S, Noeth U, Jorgensen C (2016) Adipose mesenchymal stromal cell-based therapy for severe osteoarthritis of the knee: a phase I doseescalation trial. Stem Cells Transl Med 5: 847-856.

Platas J, Guillen MI, del Caz MD, Gomar F, Mirabet V, Alcaraz MJ (2013) Conditioned media from adipose-tissue-derived mesenchymal stem cells downregulate degradative mediators induced by interleukin-1beta in osteoarthritic chondrocytes. Mediators Inflamm 2013: 357014.

Prockop DJ (2009) Repair of tissues by adult stem/ progenitor cells (MSCs): controversies, myths, and changing paradigms. Mol Ther 17: 939-946.

Prockop DJ, Oh JY (2012) Medical therapies with adult stem/progenitor cells (MSCs): a backward journey from dramatic results in vivo to the cellular and molecular explanations. J Cell Biochem 113: 14601469.

Rani S, Ryan AE, Griffin MD, Ritter T (2015) Mesenchymal stem cell-derived extracellular vesicles: toward cell-free therapeutic applications. Mol Ther 23: 812-823. 
Ren G, Zhang L, Zhao X, Xu G, Zhang Y, Roberts AI, Zhao RC, Shi Y (2008) Mesenchymal stem cellmediated immunosuppression occurs via concerted action of chemokines and nitric oxide. Cell Stem Cell 2: 141-150.

Schu S, Nosov M, O’Flynn L, Shaw G, Treacy O, Barry F, Murphy M, O'Brien T, Ritter T (2012) Immunogenicity of allogeneic mesenchymal stem cells. J Cell Mol Med 16: 2094-2103.

Tao SC, Yuan T, Zhang YL, Yin WJ, Guo SC, Zhang CQ (2017) Exosomes derived from miR-1405 p-overexpressing human synovial mesenchymal stem cells enhance cartilage tissue regeneration and prevent osteoarthritis of the knee in a rat model. Theranostics 7: 180-195.

Ter Huurne M, Schelbergen R, Blattes R, Blom A, De Munter W, Grevers LC, Jeanson J, Noël D, Casteilla L, Jorgensen C, Van Den Berg W, Van Lent PLEM (2012) Antiinflammatory and chondroprotective effects of intraarticular injection of adipose-derived stem cells in experimental osteoarthritis. Arthritis Rheum 64: 3604-3613.

Timmers L, Lim SK, Hoefer IE, Arslan F, Lai RC, van Oorschot AA, Goumans MJ, Strijder C, Sze SK, Choo A, Piek JJ, Doevendans PA, Pasterkamp G, de Kleijn DP (2011) Human mesenchymal stem cell-conditioned medium improves cardiac function following myocardial infarction. Stem Cell Res 6: 206-214.

Toh WS, Lai RC, Hui JHP, Lim SK (2016) MSC exosome as a cell-free MSC therapy for cartilage regeneration: implications for osteoarthritis treatment. Semin Cell Dev Biol 67: 56-64.

Utomo L, van Osch GJ, Bayon Y, Verhaar JA, Bastiaansen-Jenniskens YM (2016) Guiding synovial inflammation by macrophage phenotype modulation: an in vitro study towards a therapy for osteoarthritis. Osteoarthritis Cartilage 24: 1629-1638.

van Buul GM, Siebelt M, Leijs MJC, Bos PK, Waarsing JH, Kops N, Weinans H, Verhaar JAN, Bernsen MR, Van Osch GJVM (2014) Mesenchymal stem cells reduce pain but not degenerative changes in a mono-iodoacetate rat model of osteoarthritis. J Orthop Res 32: 1167-1174.

van Buul GM, Villafuertes E, Bos PK, Waarsing JH, Kops N, Narcisi R, Weinans H, Verhaar JA, Bernsen MR, van Osch GJ (2012) Mesenchymal stem cells secrete factors that inhibit inflammatory processes in short-term osteoarthritic synovium and cartilage explant culture. Osteoarthritis Cartilage 20: 11861196.

van der Kraan PM, Vitters EL, van Beuningen HM, van de Putte LB, van den Berg WB (1990) Degenerative knee joint lesions in mice after a single intra-articular collagenase injection. A new model of osteoarthritis. J Exp Pathol (Oxford) 71: 19-31.

von Bahr L, Batsis I, Moll G, Hagg M, Szakos A, Sundberg B, Uzunel M, Ringden O, Le Blanc K (2012) Analysis of tissues following mesenchymal stromal cell therapy in humans indicates limited long-term engraftment and no ectopic tissue formation. Stem Cells 30: 1575-1578.

Wang Y, Yu D, Liu Z, Zhou F, Dai J, Wu B, Zhou J, Heng BC, Zou XH, Ouyang H, Liu H (2017) Exosomes from embryonic mesenchymal stem cells alleviate osteoarthritis through balancing synthesis and degradation of cartilage extracellular matrix. Stem Cell Res Ther 8: 189.

Yuan XL, Meng HY, Wang YC, Peng J, Guo QY, Wang AY, Lu SB (2014) Bone-cartilage interface crosstalk in osteoarthritis: potential pathways and future therapeutic strategies. Osteoarthritis Cartilage 22: 1077-1089.

Zhang S, Chuah SJ, Lai RC, Hui JHP, Lim SK, Toh WS (2018) MSC exosomes mediate cartilage repair by enhancing proliferation, attenuating apoptosis and modulating immune reactivity. Biomaterials 156: 16-27.

Zhang Y, Jordan JM (2010) Epidemiology of osteoarthritis. Clin Geriatr Med 26: 355-369.

Zheng G, Huang R, Qiu G, Ge M, Wang J, Shu Q, Xu J (2018) Mesenchymal stromal cellderived extracellular vesicles: regenerative and immunomodulatory effects and potential applications in sepsis. Cell Tissue Res 374: 1-15.

Zhu Y, Wang Y, Zhao B, Niu X, Hu B, Li Q, Zhang J, Ding J, Chen Y, Wang Y (2017) Comparison of exosomes secreted by induced pluripotent stem cell-derived mesenchymal stem cells and synovial membrane-derived mesenchymal stem cells for the treatment of osteoarthritis. Stem Cell Res Ther 8: 64 .

\section{Discussion with Reviewers}

Lucienne Vonk: What would the outcome be using the secretome of not pre-activated MSCs?

Authors: Several studies show that the secretome of activated MSCs has better anti-inflammatory capacities than non-activated cells (Barrachina et al., 2017; Crop et al., 2010; van Buul et al., 2012). In the present in vitro study using human OA cartilage and synovium explants, no anti-inflammatory effects of the secretome from not pre-activated MSCs were observed. Based on these results, it is unlikely that secretome of not pre-treated MSCs would give an effect in the CIOA murine model.

Lucienne Vonk: Would extracellular vesicles perform as well as the whole secretome?

Authors: Based on the used production protocol, the MSC secretome was expected to contain both soluble factors secreted in the collecting medium as well as extracellular vesicles. Since the content of the extracellular vesicles can be different from the soluble factors present in the secretome, it is possible that the effect of isolated extracellular vesicles would not entirely match that of the entire secretome. Future studies on this topic would be necessary to elucidate this aspect. 
Lucienne Vonk: Would pre-activated MSCs perform even better?

Authors: MSCs can respond to their environment and, that way, produce the cytokines and growth factors needed at that moment at that location (Caplan and Dennis, 2006; Caplan, 2017). Pre-activating the MSCs is not necessarily better since the effect might be too strong or there might be more side-effects. Although pre-activated MSCs do not improve migration in vitro or in vivo, it is possible that injection of pre-activated MSCs would lead to a faster or a more powerful anti-inflammatory effect when injected into a joint (Leijs et al., 2017; additional reference) This might be beneficial, in particular when injected in a highly inflamed joint. It would be interesting to compare pre-activated MSCs with control MSCs in a future experiment.

Farshid Guilak: How do you differentiate between soluble factors and those present within the MSCsecreted extracellular vesicles?

Authors: The secretome used presumably contained both soluble factors and extracellular vesicles. The present set-up did not discriminate between these two. Which factors exerted the desired anti-inflammatory/regenerative effects remained unknown and it is crucial to investigate this aspect in the future to further develop this technique.
Farshid Guilak: What is the limitation of examining the effects of the secretome across species (human to mouse)?

Authors: The potency of the human MSC secretome was evaluated to increase the clinical translatability of the study. The limitation is, indeed, that some factors or receptors might not be cross-species-conserved and that the effects observed are underestimated. Moreover, the use of xenogeneic MSCs secretome might have caused more synovial inflammation, possibly since the extracellular vesicles can contain major histocompatibility complex (MHC) class I and II molecules (Zheng et al., 2018) and, thus, have reduced the analgesic effect of the MSC secretome.

\section{Additional Reference}

Leijs MJ, van Buul GM, Lubberts E, Bos PK, Verhaar JA, Hoogduijn MJ, van Osch GJ (2012) Effect of arthritic synovial fluids on the expression of immunomodulatory factors by mesenchymal stem cells: an explorative in vitro study. Front Immunol 3: 231.

Editor's note: The Scientific Editor responsible for this paper was Martin Stoddart. 